\title{
The epidemiology of polypharmacy in older adults: register-based prospective cohort study
}

This article was published in the following Dove Press journal:

Clinical Epidemiology

\author{
Lucas Morin \\ Kristina Johnell' \\ Marie-Laure Laroche ${ }^{2,3}$ \\ Johan Fastbom' \\ Jonas W Wastesson' \\ 'Aging Research Center, Karolinska \\ Institutet, Stockholm, Sweden; \\ ${ }^{2}$ University Hospital of Limoges, \\ Service de Pharmacologie, Toxicologie \\ et Pharmacovigilance, Limoges, France; \\ ${ }^{3}$ Faculté de Médecine, Université de \\ Limoges, Limoges, France
}

Correspondence: Lucas Morin Aging Research Center, Karolinska Institutet, I I 330 Stockholm, Gävlegatan 16, Sweden

Tel +46722 887094

Email lucas.morin@ki.se
Objective: Polypharmacy is the concomitant use of several drugs by a single person, and it increases the risk of adverse drug-related events in older adults. Little is known about the epidemiology of polypharmacy at the population level. We aimed to measure the prevalence and incidence of polypharmacy and to investigate the associated factors.

Methods: A prospective cohort study was conducted using register data with national coverage in Sweden. A total of 1,742,336 individuals aged $\geq 65$ years at baseline (November 1,2010) were included and followed until death or the end of the study (December 20, 2013).

Results: On average, individuals were exposed to 4.6 ( $\mathrm{SD}=4.0)$ drugs at baseline. The prevalence of polypharmacy ( $5+$ drugs) was $44.0 \%$, and the prevalence of excessive polypharmacy $(10+$ drugs) was $11.7 \%$. The incidence rate of polypharmacy among individuals without polypharmacy at baseline was 19.9 per 100 person-years, ranging from $16.8 \%$ in individuals aged $65-74$ years to $33.2 \%$ in those aged $\geq 95$ years (adjusted hazard ratio $[\mathrm{HR}]=1.49,95 \%$ confidence interval [CI] 1.42-1.56). The incidence rate of excessive polypharmacy was 8.0 per 100 person-years. Older adults using multi-dose dispensing were at significantly higher risk of developing incident polypharmacy compared with those receiving ordinary prescriptions (HR $=1.51,95 \%$ CI $1.47-1.55$ ). When adjusting for confounders, living in nursing home was found to be associated with lower risks of incident polypharmacy and incident excessive polypharmacy ( $\mathrm{HR}=0.79$ and $\mathrm{HR}=0.86, p<0.001$, respectively).

Conclusion: The prevalence and incidence of polypharmacy are high among older adults in Sweden. Interventions aimed at reducing the prevalence of polypharmacy should also target potential incident polypharmacy users as they are the ones who fuel future polypharmacy.

Keywords: drugs, older adults, polypharmacy, prescribing, medication, elderly

\section{Plain language summary}

Polypharmacy is the concomitant use of several drugs by a single person. Because polypharmacy is often the consequence of the coexistence of many chronic diseases, it is more frequent among older people than among middle-aged adults. It is potentially problematic because it can lead to serious adverse events. Previous studies have shown that, on average, polypharmacy affects between $40 \%$ and $50 \%$ of all older adults. However, little is known about how polypharmacy develops over time. In this study, about 1.7 million Swedish older adults have been followed for up to 3 years (from 2010 to 2013) through various health registers. We wanted to understand how many people were using five or more medications at the beginning of the study ("prevalence") and how rapidly the others would start using five or more medications ("incidence"). Our findings show that the prevalence of polypharmacy ( $\geq 5$ drugs) was $44.0 \%$ and the prevalence of excessive polypharmacy ( $\geq 10$ drugs) was $11.7 \%$. Moreover, among individuals who did not have polypharmacy at the beginning of the study, about $20 \%$ started polypharmacy every year, 
ranging from $16.8 \%$ in individuals aged $65-74$ years to $33.2 \%$ in those aged $\geq 95$ years. We also found that older adults using multidose dispensing were at significantly higher risk of developing incident polypharmacy compared with those receiving ordinary prescriptions.

\section{Introduction}

Polypharmacy is the concomitant use of several drugs by a single individual. ${ }^{1}$ It often reflects the coexistence of various health problems, including chronic diseases (eg, diabetes and heart failure), acute conditions (eg, infections), and symptoms (eg, pain), which accumulate with age. The application of evidence-based prescribing guidelines designed for the management of single disease to individuals with multimorbidity can result in complex drug regimens. ${ }^{2}$ The number of drugs is therefore higher among older people than among middle-aged adults. ${ }^{3}$ The use of multiple drug treatments can be clinically appropriate if they improve health and quality of life. ${ }^{4,5}$ However, it poses important challenges to clinicians because many older adults are exposed to polypharmacy beyond the point where drug therapy is beneficial. ${ }^{6-9}$

Polypharmacy increases the risk of adverse drug-related events in older adults. ${ }^{10-12}$ First, because a higher number of drugs comes with a higher risk of harmful drug-drug interactions. ${ }^{13}$ Second, because the aging process is associated with physiological changes (eg, weight loss, deterioration of liver and renal excretion, decrease of cardiac output, body composition remodeling $)^{14}$ that make older adults more prone to adverse drug reactions. ${ }^{15-18}$ Third, because the high prevalence of chronic multimorbidity in old age leads to an enhanced risk of drug-disease interactions. ${ }^{19-21}$

In high-income countries, population-based surveys and cross-sectional studies have shown that polypharmacy affects between $40 \%$ and $50 \%$ of older adults. ${ }^{22-24}$ Several risk factors have been identified, such as recent nursing home admission, number of prescribers, and frailty. ${ }^{25,26}$ Recent studies have also suggested an inverted U-shaped association between age and number of drugs, with a pronounced decline in the burden of medications after the age of 85 years. ${ }^{27}$ However, only few of these studies had the possibility to account for the confounding effect of chronic multimorbidity (confounding by indication), thus most likely overestimating the role of sociodemographic risk factors. Moreover, surprisingly little is known about incident polypharmacy, that is the development of polypharmacy over time. ${ }^{28-32}$ This lack of evidence from longitudinal, prospective studies limits our understanding of the epidemiology of polypharmacy and most likely leads to underestimating the true burden of medication use among older adults.
Using register data covering the entire population of older adults in Sweden, this study aimed to measure the prevalence of polypharmacy at baseline, to measure the incidence rate of polypharmacy over time, and finally to investigate the factors independently associated with both prevalent and incident polypharmacy.

\section{Methods}

\section{Study design and population}

We conducted a prospective, longitudinal, register-based cohort study covering all Sweden. All individuals aged $\geq 65$ years and registered as living in Sweden at baseline (November 1, 2010) were included in the study population and followed for up to 37 months (until December 20, 2013). The study design is illustrated in Figure S1.

\section{Assessment of outcomes}

Although there is currently no consensual definition of polypharmacy, ${ }^{33}$ previous studies have frequently relied on a cutoff point of $\geq 5$ medications to measure its prevalence in the older population. ${ }^{1,34,35}$ We define "polypharmacy" as the concurrent use of $\geq 5$ medications and "excessive polypharmacy" as the concurrent use of $\geq 10$ medications (ie, distinct substances according to the 5th level of Anatomical Therapeutic Chemical [ATC] classification system). Data on drug use were extracted from the Swedish Prescribed Drugs Register, which collects data about all prescription drugs delivered from pharmacies in Sweden since 2005. ${ }^{36}$ As illustrated in Figure S2, periods of drug exposure were calculated on the basis of 1) the date of drug dispensing, 2) the total amount dispensed to the patient, and 3) the prescribed daily dose. ${ }^{37,38}$

\section{Measurement of individual characteristics at baseline}

Study participants' sex and age were derived from the Total Population Register. ${ }^{39}$ Other characteristics were retrieved through record-linkage at the individual level between multiple registers with national coverage in Sweden. Highest level of education was categorized as "primary," "lower secondary," "upper secondary," and "tertiary" education in accordance with the International Standard for Classification of Education (ISCED97), and it was assessed through the Swedish Register of Education. ${ }^{40}$ Living arrangement at baseline was defined as either "community-dwelling" or "living in nursing home," using data from the Swedish National Board of Health and Welfare's Social Services 
Register. This register comprises information about care and services provided by municipalities to older adults and persons with functional impairment across the entire Sweden. Drug dispensing scheme was defined as "ordinary prescription" or "multi-dose dispensing," a dose administration aid where medications are supplied to the patients in machinepackaged disposable plastic pouches. ${ }^{41,42}$

We used a previously validated multimorbidity assessment tool to measure the overall burden of chronic diseases at baseline. ${ }^{43}$ The methodology proposed by CalderónLarrañaga et $\mathrm{al}^{43}$ allows for capturing a comprehensive set of chronic diseases that either have a long-lasting impact on older adults' autonomy and quality of life or require enduring contacts with healthcare services. This instrument is therefore well suited to describe the burden of chronic multimorbidity in our study population and to address the issue of confounding by indication. ${ }^{44}$ We identified chronic diseases by analyzing all diagnoses reported for inpatient and specialized outpatient admissions during the 3 years prior to baseline, as well as specific medications dispensed during the same period. Finally, information about the date and causes of death were obtained from the National Cause of Death Register. Time to death was thereafter categorized as "more than 12 months" or " 12 months or less," thus identifying individuals in their last year of life. Previous studies have reported a significant increase in polypharmacy near the end of life, mainly attributable to the rising prevalence of burdensome symptoms as death approaches. ${ }^{45}$

\section{Statistical analysis}

The characteristics of the study population are reported using standard descriptive statistics. Prevalence of polypharmacy and excessive polypharmacy at baseline was based on the cumulative number of prescribed medications to which older adults were exposed during the month before baseline (October 1-31, 2010). Prevalence rates were calculated as percentages of the total study population. To identify factors associated with polypharmacy at baseline, we computed a series of logistic regression models including sex, age, level of education, living arrangement, number of chronic diseases, drug dispensing scheme, and time to death as independent variables. Odds ratios (ORs) are reported with 95\% confidence intervals (CIs).

Incidence of polypharmacy and excessive polypharmacy during follow-up was calculated as the number of incident cases among older adults who were not exposed to $\geq 5$ or $\geq 10$ prescription drugs during the 6-month washout period before baseline (Figure 1). Incidence rates are reported as the number of individuals who had a first exposure to $\geq 5$ or $\geq 10$ medications for 100 person-years, thus reflecting single-failure incidence. Contributing time was considered from baseline until 1) the first occurrence of polypharmacy, 2) death, or 3 ) the end of the study. We used Cox proportional hazard regression models to investigate the factors associated with a higher risk of developing incident polypharmacy. Hazard ratios (HRs) are adjusted for different sets of confounders and are reported with 95\% CI. Finally, we calculated and plotted the Kaplan-Meier failure function of polypharmacy by age and by number of drugs at baseline. This function represents the cumulative fraction of individuals experiencing incident polypharmacy throughout the follow-up period. Sensitivity analyses were performed to ensure the reliability of our findings. First, we calculated the prevalence of polypharmacy at baseline while removing antiinfectives for systemic use from the total number of drugs. This allowed for estimating the contribution of short-term antibiotic drug treatments to polypharmacy. Second, we calculated polypharmacy based on the number of therapeutic subgroups $\left(2^{\text {nd }}\right.$ level of the ATC classification) at baseline instead of the number of chemical substances ( $5^{\text {th }}$ level of the ATC classification), in order to account for the fact that older adults may switch between two medications from the same class.

\section{Ethical approval}

Data were anonymized, and the Regional Ethical Review Board in Stockholm approved the study (2013/1941-31/3 and 2015/1319-32).

\section{Results}

\section{Characteristics of the study population}

Overall, 1,742,336 individuals aged $\geq 65$ years were included at baseline, accounting for $99.1 \%$ of the total number of older adults living in Sweden in November 2010. Mean age was 75 years (SD, 7.8), with $15 \%$ of study participants aged $\geq 85$ years. As shown in Table 1, almost one fifth of individuals had completed a higher education degree, 90,007 (5.2\%) were living in nursing homes and $17 \%$ had at least five chronic diseases. A total of 242,765 (13.9\%) study participants died during the 3-year follow-up, including 76,428 (4.4\%) who died within 12 months. Older adults free of polypharmacy at baseline $(n=812,824)$ were younger, healthier, and less often institutionalized than average. The characteristics of older adults free of excessive polypharmacy at baseline $(\mathrm{n}=1,438,437)$ are reported in Table S1. 


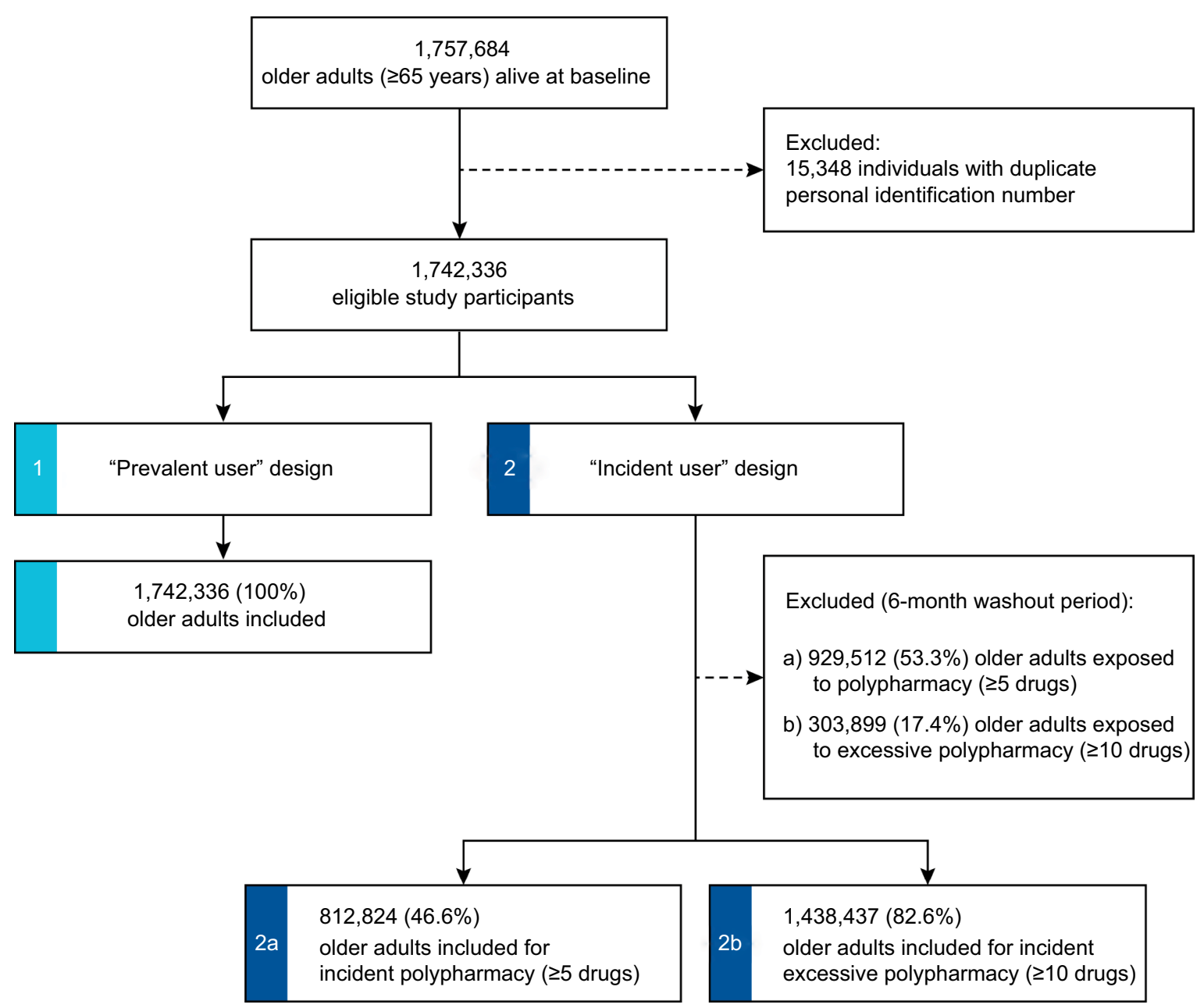

Figure I Patient inclusion flowchart.

\section{Prevalence of polypharmacy at baseline}

At baseline, older adults were exposed to 4.6 different drugs on average (SD, 4.0), ranging from 4.4 drugs among those living in the community to 8.2 in nursing homes (Table 2). The number of drugs was strongly correlated with the number of chronic conditions. Hence, each additional chronic disease was associated with a 0.95 increase in the number of drugs (95\% CI 0.94-0.96). Overall, the prevalence of polypharmacy ( $\geq 5$ drugs) and excessive polypharmacy ( $\geq 10$ drugs) was $44.0 \%$ and $11.7 \%$, respectively. Detailed estimates with different cutoff points are available in Table S2. At baseline, women sex was independently associated with a $28 \%$ increased likelihood of being exposed to polypharmacy. People living in nursing homes were more often exposed to polypharmacy than those living in the community. However, after controlling for possible confounders, living in nursing home was found to be associated with decreased odds of being exposed to polypharmacy
$(\mathrm{OR}=0.80,95 \%$ CI $0.78-0.82)$. As shown in Table S3, it was associated with a modest increase in the likelihood to be exposed to excessive polypharmacy $(\mathrm{OR}=1.07$, 95\% CI 1.05-1.09). In a linear regression model, living in institution was independently associated with a $0.15(95 \%$ CI 0.12-0.18) reduction of the total number of prescribed drugs at baseline (data not shown). In contrast, multi-dose dispensing led to significantly higher prevalence rates of polypharmacy and excessive polypharmacy, both among community-dweller and among institutionalized older adults (Figures $\mathrm{S} 3$ and $\underline{\mathrm{S} 4}$ ). Sensitivity analyses showed that the prevalence of polypharmacy at baseline remained stable after removing systemic antiinfectives from the total number of drugs (Table S4) or when considering therapeutic subgroups instead of chemical substances (Figure S5). Moreover, the mean number of drugs at baseline was mostly fueled by medications of the cardiovascular and nervous systems (Figure S6). 
Table I Characteristics of the study population at baseline

\begin{tabular}{lll}
\hline Characteristics & Full cohort & $\begin{array}{l}\text { Polypharmacy- } \\
\text { free at baseline }\end{array}$ \\
\hline $\begin{array}{ll}\text { Total, N } \\
\text { Sex }\end{array}$ & $1,742,336$ & 812,824 \\
$\quad$ Men & $782,503(44.9 \%)$ & $397,414(48.9 \%)$ \\
Women & $959,833(55.1 \%)$ & $415,410(51.1 \%)$ \\
Age, years & & \\
Mean (SD) & $75.1(7.8)$ & $72.8(7.0)$ \\
N (\%) & & \\
$\quad 65-74$ & $936,163(53.7 \%)$ & $542,300(66.7 \%)$ \\
$\quad 75-84$ & $551,710(31.7 \%)$ & $204,892(25.2 \%)$ \\
$\quad 85-94$ & $237,086(13.6 \%)$ & $61,306(7.5 \%)$ \\
$\quad \geq 95$ & $17,377(1.0 \%)$ & $4,326(0.5 \%)$ \\
Living arrangement & & \\
Community & $1,652,329(94.8 \%)$ & $801,516(98.6 \%)$ \\
Nursing home & $90,007(5.2 \%)$ & $11,308(1.4 \%)$ \\
Number of chronic diseases & & \\
Mean (SD) & $2.4(2.4)$ & $1.0(1.3)$ \\
N (\%) & & \\
$\quad 0$ & $432,537(24.8 \%)$ & $368,898(45.4 \%)$ \\
I & $373,378(21.4 \%)$ & $228,104(28.1 \%)$ \\
2 & $284,124(16.3 \%)$ & $119,011(14.6 \%)$ \\
3 & $206,689(11 . \%)$ & $55,414(6.8 \%)$ \\
4 & $148,141(8.5 \%)$ & $24,419(3 \%)$ \\
$\quad \geq 5$ & $297,467(17.1 \%)$ & $16,978(2.1 \%)$ \\
Drug dispensing scheme & & \\
Ordinary prescriptions & $1,595,776(91.6 \%)$ & $801,598(98.6 \%)$ \\
Multi-dose dispensing & $146,560(8.4 \%)$ & $11,226(1.4 \%)$ \\
Time to death & & \\
$>12$ months & $1,665,908(95.6 \%)$ & $799,695(98.4 \%)$ \\
$\leq 12$ months & $76,428(4.4 \%)$ & $13,129(1.6 \%)$ \\
Level of education ${ }^{b}$ & & \\
Lower secondary education & $117,615(6.9 \%)$ & $55,609(6.8 \%)$ \\
Upper secondary education & $618,285(36.3 \%)$ & $298,150(36.7 \%)$ \\
\hline Noter education & $326,724(19.2 \%)$ & $177,706(21.9 \%)$ \\
\hline
\end{tabular}

Notes: Individuals who were not exposed to polypharmacy during the 6-month washout period before baseline ("incident user" design). 'Missing values for the level of education: $n=37,765(2.2 \%)$

\section{Incidence of polypharmacy during follow-up}

As reported in Table 3, among the 812,824 older adults who were not exposed to polypharmacy during the 6-month washout period prior to baseline, the overall incidence rate of polypharmacy was 19.9 per 100 person-years. We found substantial variation across age groups in the population, ranging from 16.8 per 100 person-years among people aged 65-74 years to 33.2 per 100 person-years among those aged $\geq 95$ years. The overall incidence of excessive polypharmacy was 8.0 per 100 person-years (Table S5). Living in nursing home seemed to be associated with an increased risk of developing incident polypharmacy and excessive polypharmacy over time ( $\mathrm{HR}=1.05$ and $1.22, p<0.001$, respectively) when accounting for the confounding effect of sex, age, and multimorbidity. However, once multivariate models were further adjusted to control for education, multi-dose dispensing, and time-to-death, we found a negative association between living in institution and risk of incident polypharmacy ( $\mathrm{HR}=0.79$, 95\% CI 0.77-0.82).

The cumulative incidence of polypharmacy increased with age (Figure 2A). At 1 year of follow-up, the risk of having developed polypharmacy varied from $20 \%$ among individuals aged $65-74$ years to $34 \%$ among those aged $\geq 95$ years. At 3 years, this risk varied from $53 \%$ to $87 \%$, respectively. Similarly, age differences were observed for the incidence of excessive polypharmacy (Figure S7). The incidence rate of polypharmacy was higher for older adults with four drugs at baseline than for those who were exposed to fewer medications (Figure 2B; Table S6). In addition, sensitivity analyses showed that the incidence rate of polypharmacy was lower when we considered only episodes of polypharmacy that lasted for $\geq 3$ months ( 11.5 per 100 person-years). Detailed results are presented in Table S7 and Figure S8.

\section{Discussion}

Three main findings stem from this large, prospective cohort study in Sweden. First, at baseline, $44 \%$ of older adults are exposed to polypharmacy ( $\geq 5$ drugs) and $12 \%$ are exposed to excessive polypharmacy ( $\geq 10$ drugs). Second, during follow-up, the incidence rate of polypharmacy was 20 per 100 person-years and that of excessive polypharmacy was 8 per 100 person-years. Third, higher age, more chronic diseases, and multi-dose dispensing led to a more frequent exposure to polypharmacy (both at baseline and throughout the follow-up period). While adjusting for these risk factors, living in nursing home was associated with a $20 \%$ reduction of the hazard of developing incident polypharmacy.

The proportion of older adults in Sweden exposed to polypharmacy $(44 \%)$ and excessive polypharmacy $(12 \%)$ is well within the range of previous reports. In the United States, data from the National Health and Nutrition Examination Survey indicate that $39 \%$ of community-dwelling older adults were exposed to $\geq 5$ prescription drugs in 2012..,22 The prevalence of polypharmacy is typically higher in the nursing home setting. ${ }^{25,46,47}$ In a study conducted among nursing home residents in eight European countries, $74 \%$ were exposed to polypharmacy and $24 \%$ to excessive polypharmacy (compared with $82 \%$ and $36 \%$ in the present study). ${ }^{48}$ Our findings thus confirm that the prevalence of polypharmacy in Sweden is comparable to that of other high-income countries. 
Table 2 Number of prescribed drugs and prevalence of polypharmacy at baseline

\begin{tabular}{|c|c|c|c|c|}
\hline \multirow[t]{2}{*}{ Baseline characteristics } & \multicolumn{2}{|c|}{ Number of drugs } & \multicolumn{2}{|c|}{ Polypharmacy ( $\geq 5$ drugs) } \\
\hline & Mean (SD) & Median (IQR) & $\mathbf{N}(\%)$ & Adjusted OR $(95 \% \mathrm{Cl})^{2}$ \\
\hline Total cohort & $4.6(4.0)$ & $4(I-7)$ & $766,900(44.0)$ & \\
\hline \multicolumn{5}{|l|}{ Sex } \\
\hline Men & $4.2(3.8)$ & $4(I-6)$ & $315,970(40.4)$ & 1 \\
\hline Women & $4.9(4.1)$ & $4(2-7)$ & $450,930(47.0)$ & $1.28(1.27-1.29)$ \\
\hline \multicolumn{5}{|l|}{ Age, years } \\
\hline $65-74$ & $3.6(3.6)$ & $3(1-5)$ & $307,351(32.8)$ & 1 \\
\hline $75-84$ & $5.3(4.0)$ & $5(2-8)$ & $292,515(53.0)$ & $1.43(1.42-1.44)$ \\
\hline $85-94$ & $6.4(4.0)$ & $6(3-9)$ & $155,395(65.5)$ & $1.62(1.60-1.64)$ \\
\hline$\geq 95$ & $6.4(3.9)$ & $6(4-9)$ & II,639 (67.0) & $1.35(1.30-1.42)$ \\
\hline \multicolumn{5}{|l|}{ Living arrangement } \\
\hline Community & $4.4(3.8)$ & $4(I-7)$ & $693,395(42.0)$ & 1 \\
\hline Nursing home & $8.2(4.2)$ & $8(5-11)$ & $73,505(81.7)$ & $0.80(0.78-0.82)$ \\
\hline \multicolumn{5}{|l|}{ Number of chronic diseases } \\
\hline 0 & I.6 (2.0) & I (0-3) & $42,095(9.7)$ & 1 \\
\hline 1 & $3.3(2.7)$ & $3(1-5)$ & $106,705(28.6)$ & $3.35(3.31-3.39)$ \\
\hline 2 & $4.5(3.0)$ & $4(2-6)$ & $128,880(45.4)$ & $6.62(6.53-6.70)$ \\
\hline 3 & $5.6(3.2)$ & $5(3-8)$ & $123,819(59.9)$ & II.58 (II.42-I.74) \\
\hline 4 & $6.6(3.4)$ & $6(4-9)$ & $105,886(7 \mid .5)$ & $18.88(18.58-19.17)$ \\
\hline$\geq 5$ & $9.0(4.2)$ & $9(6-12)$ & $259,515(87.2)$ & $47.33(46.62-48.06)$ \\
\hline \multicolumn{5}{|l|}{ Drug dispensing scheme } \\
\hline Ordinary prescriptions & $4.2(3.7)$ & $4(I-6)$ & $637,856(40.0)$ & 1 \\
\hline Multi-dose dispensing & $8.9(4.0)$ & $9(6-11)$ & $129,044(88.0)$ & $5.20(5.08-5.32)$ \\
\hline \multicolumn{5}{|l|}{ Time to death } \\
\hline$>12$ months & $4.4(3.9)$ & $4(I-7)$ & $709,098(42.6)$ & 1 \\
\hline$\leq 12$ months & $7.9(4.6)$ & $8(5-11)$ & $57,802(75.6)$ & $1.27(1.24-1.30)$ \\
\hline \multicolumn{5}{|l|}{ Level of education } \\
\hline Primary education & $5.1(4.1)$ & $5(2-8)$ & $323,069(50.3)$ & I \\
\hline Lower secondary education & $4.5(4.0)$ & $4(I-7)$ & $50,924(43.3)$ & $0.89(0.87-0.90)$ \\
\hline Upper secondary education & $4.4(3.9)$ & $4(I-7)$ & $261,378(42.3)$ & $0.88(0.87-0.89)$ \\
\hline Higher education & $3.9(3.7)$ & $3(1-6)$ & I I 6,398 (35.6) & $0.72(0.72-0.73)$ \\
\hline
\end{tabular}

Note: ${ }^{2}$ Logistic regression model including all presented covariates as independent variables. Because of missing values for the level of education ( $\left.\mathrm{n}=37,765\right)$, adjusted odds ratios (OR) and $95 \%$ confidence intervals $(\mathrm{Cl})$ are calculated for a subset of $1,704,57 \mathrm{I}$ individuals $(97.8 \%$ of total).

Abbreviation: IQR, interquartile range.

Few studies have investigated the incidence of polypharmacy. ${ }^{28,30,49}$ By casting light on the development of polypharmacy over time and by looking at the risk factors associated with future exposure to polypharmacy, we believe that the present study fills an important knowledge gap. Within the population of older adults free of polypharmacy at the time of inclusion, about $20 \%$ of individuals develop polypharmacy during the following year, thus suggesting that most of the older adults will be affected by polypharmacy during their remaining lifespan. This finding has important implications. During the past decade, several interventions aimed at reducing polypharmacy have been implemented but have been proven mostly unsuccessful. ${ }^{5,50,51}$ We believe that future interventions targeting polypharmacy should not only focus on patients that are already exposed to polypharmacy but also include prevention strategies to reduce the number of incident cases.
The number of chronic diseases and the use of multi-dose dispensing were both associated with a higher risk of both prevalent and incident polypharmacy. Correlation between the number of chronic diseases and the number of prescribed drugs is expected, which supports the hypothesis that polypharmacy is for the most part the reflection of chronic multimorbidity. ${ }^{52}$ However, the magnitude of the discrepancy between older adults with ordinary prescriptions and those with multi-dose dispensing raises concern. Although establishing causation is beyond the scope of our study, the strong and consistent association between drug dispensing and risk of polypharmacy during follow-up supports the notion that multi-dose dispensing has an inflationary effect on the number of drugs prescribed to older adults. ${ }^{41,53}$ We hypothesize that automated multi-dose dispensing promotes routine-like prescribing and may discourage physicians and pharmacists to regularly verify the adequateness of drug therapy and to 
Table 3 Incidence of polypharmacy ( $\geq 5$ drugs) during follow-up

\begin{tabular}{|c|c|c|c|c|c|}
\hline \multirow[t]{3}{*}{ Baseline characteristics } & \multirow{2}{*}{$\begin{array}{l}\text { Person- } \\
\text { years }^{\mathrm{a}}\end{array}$} & \multirow[t]{2}{*}{ Failures $^{\mathbf{b}}$} & \multirow[t]{2}{*}{ Incidence rate } & \multicolumn{2}{|l|}{ Hazard ratio } \\
\hline & & & & Model Ic & \multirow{2}{*}{$\begin{array}{l}{\text { Model } 2^{d}}^{\text {HR }(95 \% \mathrm{Cl})}\end{array}$} \\
\hline & $\mathbf{N}$ & $\mathbf{N}$ & Per 100 person-years & HR (95\%Cl) & \\
\hline Total cohort & $1,823,560$ & 363,704 & $19.9(19.9-20.0)$ & - & - \\
\hline \multicolumn{6}{|l|}{ Sex } \\
\hline Men & 906,267 & $|7|, 454$ & $18.9(\mid 8.8-19.0)$ & 1 & 1 \\
\hline Women & 917,294 & 192,250 & $21.0(20.9-21.1)$ & $1.08(1.08-1.09)$ & $1.09(1.08-1.09)$ \\
\hline \multicolumn{6}{|l|}{ Age, years } \\
\hline $65-74$ & $\mathrm{I}, 288,850$ & 216,145 & $16.8(16.7-16.8)$ & I & I \\
\hline $75-84$ & 421,492 & 108,868 & $25.8(25.7-26.0)$ & $1.36(1.35-1.37)$ & $1.34(1.33-1.35)$ \\
\hline $85-94$ & $|06,78|$ & 36,552 & $34.2(33.9-34.6)$ & $1.64(1.62-1.65)$ & $1.55(1.53-1.57)$ \\
\hline$\geq 95$ & 6,437 & 2,139 & $33.2(31.8-34.7)$ & $1.60(1.53-1.67)$ & $1.49(1.42-1.56)$ \\
\hline \multicolumn{6}{|l|}{ Living arrangement } \\
\hline Community & $1,809,158$ & 357,421 & $19.8(19.7-19.8)$ & 1 & 1 \\
\hline Nursing Home & 14,402 & 6,283 & $43.6(42.6-44.7)$ & $1.05(1.03-1.08)$ & $0.79(0.77-0.82)$ \\
\hline \multicolumn{6}{|l|}{ Number of chronic diseases } \\
\hline 0 & 942,319 & 117,259 & $12.4(\mid 2.4-12.5)$ & $\mathrm{I}$ & 1 \\
\hline 1 & 498,721 & 109,934 & $22.0(21.9-22.2)$ & $1.69(1.68-1.70)$ & $1.67(1.65-1.69)$ \\
\hline 2 & 229,403 & 69,383 & $30.2(30.0-30.5)$ & $2.23(2.21-2.26)$ & $2.20(2.18-2.22)$ \\
\hline 3 & 94,313 & 36,702 & $38.9(38.5-39.3)$ & $2.79(2.75-2.82)$ & $2.73(2.70-2.76)$ \\
\hline 4 & 36,966 & $|7,50|$ & $47.3(46.6-48.0)$ & $3.29(3.23-3.34)$ & $3.20(3.15-3.25)$ \\
\hline$\geq 5$ & 21,838 & 12,925 & $59.2(58.2-60.2)$ & $3.92(3.85-3.99)$ & $3.78(3.7 I-3.85)$ \\
\hline \multicolumn{6}{|l|}{ Drug dispensing scheme } \\
\hline Ordinary prescriptions & $1,809,966$ & 356,022 & $19.7(19.6-19.7)$ & & I \\
\hline Multi-dose dispensing & 13,594 & 7,82 & $56.5(55.3-57.8)$ & & $1.5 \mathrm{I}(1.47-1.55)$ \\
\hline \multicolumn{6}{|l|}{ Time to death } \\
\hline$>12$ months & $1,818,137$ & 358,458 & $19.7(19.7-19.8)$ & & I \\
\hline$\leq 12$ months & 5,423 & 5,246 & $96.7(94.1-99.4)$ & & $2.4 \mathrm{I}(2.34-2.48)$ \\
\hline \multicolumn{6}{|l|}{ Level of education } \\
\hline Primary education & 564,985 & 125,438 & $22.2(22.1-22.3)$ & & I \\
\hline Lower secondary education & 125,317 & 24,655 & $19.7(19.4-19.9)$ & & $0.97(0.96-0.98)$ \\
\hline Upper secondary education & 673,700 & 132,484 & $19.7(19.6-19.8)$ & & $0.98(0.97-0.99)$ \\
\hline Higher education & 413,352 & 74,044 & $17.9(17.8-18.0)$ & & $0.92(0.91-0.93)$ \\
\hline
\end{tabular}

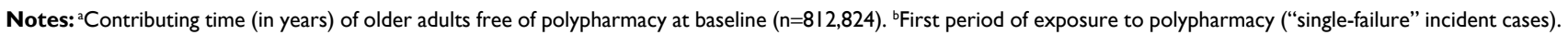
'Cox proportional hazard regression model including sex, age, and number of chronic diseases. ${ }^{\circ}$ Cox proportional hazard regression model including all covariates presented in the table. Because of missing values for the level of education $(n=19,48 \mathrm{I} ; 2.4 \%)$, results from model I are based on a subset of $793,343(97.6 \%)$ individuals.

Abbreviations: $\mathrm{HR}$, hazard ratio; $\mathrm{Cl}$, confidence intervals.

deprescribe medications that show little benefit. Multi-dose dispensing represents a potential target for future interventions aiming at reducing inappropriate polypharmacy among older adults. We also found, unexpectedly, that living in institution was independently associated with lower odds of being exposed to polypharmacy at baseline and with a decreased risk of developing incident polypharmacy over time. This suggests that living in a nursing home might not be an independent risk factor for polypharmacy when considering the burden of morbidity among institutionalized individuals, something that earlier studies could often not account for. ${ }^{25}$

In Sweden, we found that the likelihood of being exposed to polypharmacy remained stable after the age of 85 years, not only at baseline but also during follow-up. This contradicts the findings from a nationwide study in Italy, which reported that the burden of medications increases steeply until the age of 85-90 years and then declines substantially. ${ }^{27}$ This absence of decline in drug use at the very old ages has also been found in a longitudinal cohort of Danish nonagenarians ${ }^{29}$ and suggests that drug therapy continues to be extensive also among the oldest old, when a careful approach to drug prescribing is warranted.

Although prescription of multiple drugs can be clinically justified and is not necessarily inappropriate, it presents significant risks as it increases the probability of adverse drugrelated events. Moreover, polypharmacy comes with the risk of "overtreatment," a situation that arises if the prescribed medications have no clinically significant benefit during the patient's lifetime or if the risk of harm associated with the receipt of an additional medication outweighs the overall 
A By age at baseline

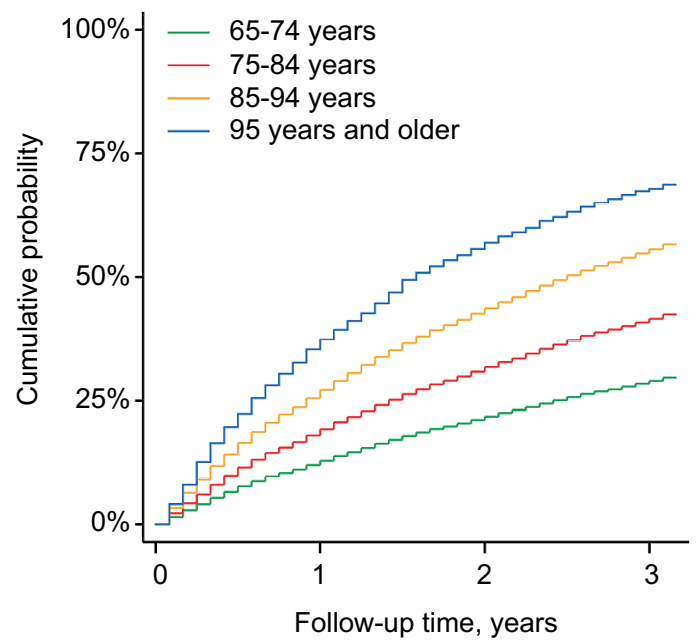

B By number of drugs at baseline

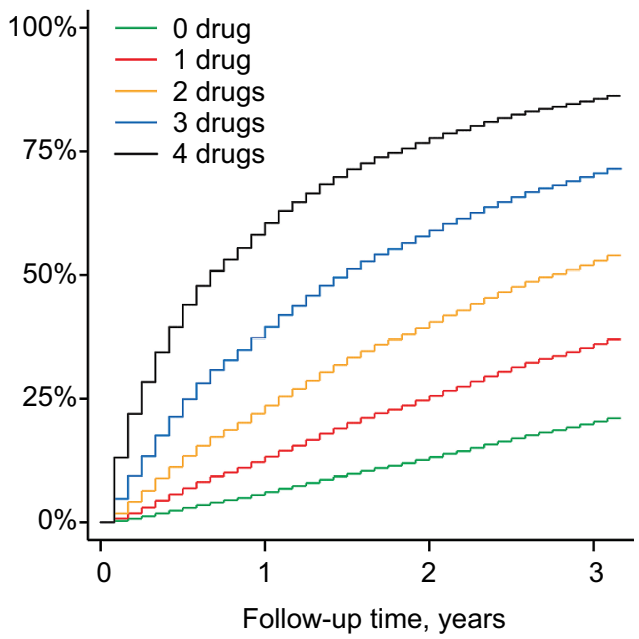

Figure 2 Cumulative incidence of polypharmacy ( $\geq 5$ drugs) during follow-up.

Notes: Curves represent the Kaplan-Meier failure function over time. (A) Estimates across age groups are adjusted for sex, living arrangement, number of chronic diseases at baseline, drug dispensing scheme, level of education, and time to death. (B) Kaplan-Meier estimator is unadjusted and reflects the crude association between the number of drugs at baseline and the cumulative probability of developing incident polypharmacy during the 3-year follow-up.

benefit of the treatments that the patient already receives. ${ }^{54-56}$ Prescribers should therefore be encouraged to reconsider the continuation of preventive drugs when the time needed for the treatment to achieve a clinically meaningful outcome is longer than the patients' remaining life expectancy. ${ }^{57}$ In that regard, our results are in line with previous studies questioning the burden of medications during the last months of life of older adults with life-limiting conditions. ${ }^{45,58}$

This study relies on routinely collected data with full-population coverage in Sweden. To the best of our knowledge, it is the first time that such a large cohort of older adults ( 1.7 million individuals) is assembled and that their drug utilization is followed month-by-month for up to 3 years. Moreover, we provide estimates of the prevalence and incidence of polypharmacy not only among communitydwellers but also among institutionalized older adults. The calculation of drug exposure at monthly intervals offers the opportunities to study the development of polypharmacy over time with a fine-grained temporal resolution. Finally, the use of validated methods to measure the burden of chronic diseases reduces the bias of confounding by indication. However, the findings reported in this article should be interpreted with caution, in light of several limitations. First, as it is often the case with register-based studies, important clinical and biological parameters were unavailable (eg, renal function, body mass index, and frailty), thus precluding a more detailed analysis of the clinical risk profile of older adults with and without polypharmacy. Second, our 3-year follow-up may not be sufficient to uncover the long-term development of polypharmacy. Third, we only investigated the total number of prescribed drugs. Future studies should investigate how specific drug classes contribute to the development of incident polypharmacy over time. Fourth, it may be argued that positioning the baseline in November could lead to observing the effect of seasonality rather than the true incidence of polypharmacy. This hypothesis is however not supported by our data (Figure S9). In fact, drug classes that are most affected by seasonality (eg, antibiotics and cough suppressants) have a low contribution to the total burden of prescription drugs, which explains that the prevalence of polypharmacy is stable across seasons. Fifth, the Swedish Prescribed Drugs Register collects only data about prescription drugs delivered from community pharmacies. This includes drugs prescribed by hospital physicians working in inpatient or specialized outpatient care facilities dispensed by community pharmacies. However, it does not include drug regimens administered within the hospital setting. Also, over-the-counter medications are not included, which may lead to a slight underestimation of the number of drugs used by older adults. Based on the wholesale data, we estimate that over $86 \%$ of all defined daily doses used in Sweden are accounted for in our study. Also, by calculating single-failure incidence rates, we only consider the first new episode of polypharmacy and we provide no information about the duration of each episode. Further research is therefore warranted to study the 
"chronicity" of polypharmacy. Finally, it should be noted that this study does not assess the appropriateness of treatment with multiple medications on an individual level.

\section{Conclusion}

In this nationwide, longitudinal study of 1.7 million people, we found that nearly half of older adults were exposed to polypharmacy at baseline and that about $20 \%$ of the remaining half experienced polypharmacy during the first year of follow-up. First, these findings show that cross-sectional studies underestimate the true burden of polypharmacy in old age. Second, it suggests that to reduce the prevalence of polypharmacy, interventions should not only focus on patients who are already exposed to polypharmacy but also include prevention strategies to reduce the number of incident cases as they are the ones who fuel future polypharmacy.

\section{Availability of data and materials}

Individual data from the Swedish Prescribed Drugs Register cannot be made publicly available. Interested researchers can access the aggregated data from the Swedish Prescribed Drugs Register (www.socialstyrelsen.se/statistik/ statistikdatabas/lakemedel).

\section{Acknowledgments}

The study reported in this article has been presented at the $21^{\text {th }}$ IAGG World Congress in San Francisco (2017).

This work was supported by a grant from the Swedish Research Council for Health, Working Life and Welfare and the Swedish Research Council. The funders had no role in study design, data collection and analysis, decision to publish, or preparation of the manuscript.

\section{Author contributions}

LM and JWW conceived and designed the study, performed the statistical analysis, interpreted the data, and drafted and critically revised the manuscript. JF acquired the data and critically revised the manuscript. KJ and MLL obtained funding, provided supervision, interpreted the data, and critically revised the manuscript. All the authors gave approval for the final version of the manuscript and agree to be accountable for all aspects of the work.

\section{Disclosure}

The authors report no conflicts of interest in this work.

\section{References}

1. Fincke BG, Snyder K, Cantillon C, et al. Three complementary definitions of polypharmacy: methods, application and comparison of findings in a large prescription database. Pharmacoepidemiol Drug Saf. 2005; 14(2):121-128.

2. Wallace E, Salisbury C, Guthrie B, Lewis C, Fahey T, Smith SM Managing patients with multimorbidity in primary care. $\mathrm{Br}$ Med $\mathrm{J}$. 2015;350:h176.

3. Kantor ED, Rehm CD, Haas JS, Chan AT, Giovannucci EL. Trends in prescription drug use among adults in the United States from 1999-2012. JAMA. 2015;314(17):1818.

4. Wise J. Polypharmacy: a necessary evil. BMJ. 2013;347:f7033.

5. Duerden M, Avery T, Payne R. Polypharmacy and Medicines Optimisation. Making It Safe and Sound. London: The King's Fund; 2013.

6. Scott IA, Hilmer SN, Reeve E, et al. Reducing inappropriate polypharmacy: the process of deprescribing. JAMA Intern Med. 2015;175(5): 827-834.

7. Vetrano DL, Tosato M, Colloca G, et al. Polypharmacy in nursing home residents with severe cognitive impairment: results from the SHELTER Study. Alzheimers Dement. 2013;9(5):587-593.

8. Jansen J, Naganathan V, Carter SM, et al. Too much medicine in older people? Deprescribing through shared decision making. $\mathrm{Br}$ Med $\mathrm{J}$. 2016;2893:i2893.

9. Payne RA, Avery AJ. Polypharmacy: one of the greatest prescribing challenges in general practice. Br J Gen Pract. 2011;61(583): $83-84$.

10. Davies EA, O'Mahony MS. Adverse drug reactions in special populations - the elderly. Br J Clin Pharmacol. 2015;80(4):796-807.

11. Wauters M, Elseviers M, Vaes B, et al. Too many, too few, or too unsafe? Impact of inappropriate prescribing on mortality, and hospitalization in a cohort of community-dwelling oldest old. Br J Clin Pharmacol. 2016;82(5):1382-1392.

12. Atkin PA, Veitch PC, Veitch EM, Ogle SJ. The epidemiology of serious adverse drug reactions among the elderly. Drugs Aging. 1999;14(2):141-152.

13. Johnell K, Klarin I. The relationship between number of drugs and potential drug-drug interactions in the elderly: a study of over 600,000 elderly patients from the Swedish Prescribed Drug Register. Drug Saf. 2007;30(10):911-918.

14. Santanasto AJ, Goodpaster BH, Kritchevsky SB, et al. Body composition remodeling and mortality: the health aging and body composition study. J Gerontol A Biol Sci Med Sci. 2017;72(4):513-519.

15. Shi S, Mörike K, Klotz U. The clinical implications of ageing for rational drug therapy. Eur J Clin Pharmacol. 2008;64(2):183-199.

16. Sera LC, McPherson ML. Pharmacokinetics and pharmacodynamic changes associated with aging and implications for drug therapy. Clin Geriatr Med. 2012;28(2):273-286.

17. Hubbard RE, O'Mahony MS, Woodhouse KW. Medication prescribing in frail older people. Eur J Clin Pharmacol. 2013;69(3):319-326.

18. Mangoni A, Jackson SHD. Age-related changes in pharmacokinetics and pharmacodynamics: basic principles and practical applications. $\mathrm{Br}$ J Clin Pharmacol. 2004;57(1):6-14.

19. Marengoni A, Winblad B, Karp A, Fratiglioni L. Prevalence of chronic diseases and multimorbidity among the elderly population in Sweden. Am J Public Health. 2008;98(7):1198-1200.

20. Fortin M, Stewart M, Poitras ME, Almirall J, Maddocks H. A systematic review of prevalence studies on multimorbidity: toward a more uniform methodology. Ann Fam Med. 2012;10(2):142-151.

21. Mallet L, Spinewine A, Huang A. The challenge of managing drug interactions in elderly people. Lancet. 2007;370(9582):185-191.

22. Charlesworth CJ, Smit E, Lee DSH, Alramadhan F, Odden MC. Polypharmacy among adults aged 65 years and older in the United States: 1988-2010. J Gerontol A Biol Sci Med Sci. 2015;70(8): 989-995. 
23. Guthrie B, Makubate B, Hernandez-Santiago V, Dreischulte T. The rising tide of polypharmacy and drug-drug interactions: population database analysis 1995-2010. BMC Med. 2015;13(1):74.

24. Hajjar ER, Cafiero AC, Hanlon JT. Polypharmacy in elderly patients. Am J Geriatr Pharmacother. 2007;5(4):345-351.

25. Jokanovic N, Tan ECK, Dooley MJ, Kirkpatrick CM, Bell JS. Prevalence and factors associated with polypharmacy in long-term care facilities: a systematic review. JAm Med Dir Assoc. 2015;16(6):535.e1-535.e12.

26. O'Dwyer M, Peklar J, McCallion P, McCarron M, Henman MC. Factors associated with polypharmacy and excessive polypharmacy in older people with intellectual disability differ from the general population: a cross-sectional observational nationwide study. BMJ Open. 2016;6(4): $\mathrm{e} 010505$.

27. Onder G, Marengoni A, Russo P, et al. Advanced age and medication prescription: more years, less medications? A nationwide report from the Italian Medicines Agency. J Am Med Dir Assoc. 2015;17(2):168-172.

28. Veehof L, Stewart R, Haaijer-Ruskamp F, Jong BM. The development of polypharmacy. A longitudinal study. Fam Pract. 2000;17(3):261-267.

29. Wastesson JW, Oksuzyan A, von Bornemann Hjelmborg J, Christensen K. Changes in drug use and polypharmacy after the age of 90: a longitudinal study of the Danish 1905 cohort. J Am Geriatr Soc. 2017;65(1):160-164.

30. Franchi C, Marcucci M, Mannucci PM, et al. Changes in clinical outcomes for community-dwelling older people exposed to incident chronic polypharmacy: a comparison between 2001 and 2009. Pharmacoepidemiol Drug Saf. 2016;25(2):204-211.

31. Abolhassani N, Castioni J, Marques-Vidal P, Vollenweider P, Waeber G. Determinants of change in polypharmacy status in Switzerland: the population-based CoLaus study. Eur J Clin Pharmacol. 2017;73(9):1187-1194.

32. Jyrkkä J, Vartiainen L, Hartikainen S, Sulkava R, Enlund H. Increasing use of medicines in elderly persons: a five-year follow-up of the Kuopio 75+Study. Eur J Clin Pharmacol. 2006;62(2):151-158.

33. Gnjidic D, Hilmer SN, Blyth FM, et al. Polypharmacy cutoff and outcomes: five or more medicines were used to identify communitydwelling older men at risk of different adverse outcomes. $J$ Clin Epidemiol. 2012;65(9):989-995.

34. Mortazavi SS, Shati M, Keshtkar A, Malakouti SK, Bazargan M, Assari S. Defining polypharmacy in the elderly: a systematic review protocol. BMJ Open. 2016;6(3): $\mathrm{e} 010989$.

35. Sirois C, Laroche M-L, Guénette L, Kröger E, Cooper D, Émond V. Polypharmacy in multimorbid older adults: protocol for a systematic review. Syst Rev. 2017;6(1):104.

36. Wettermark B, Hammar N, Fored CM, et al. The new Swedish Prescribed Drug Register - opportunities for pharmacoepidemiological research and experience from the first six months. Pharmacoepidemiol Drug Saf. 2007;16(7):726-735.

37. Lau HS, De Boer A, Beuning KS, Porsius A. Validation of pharmacy records in drug exposure assessment. J Clin Epidemiol. 1997;50(5):619-625.

38. Johnell K, Fastbom J. Comparison of prescription drug use between community-dwelling and institutionalized elderly in Sweden. Drugs Aging. 2012;29(9):751-758.

39. Ludvigsson JF, Almqvist C, Bonamy A-KE, et al. Registers of the Swedish total population and their use in medical research. Eur J Epidemiol. 2016;31(2):125-136.

Clinical Epidemiology

\section{Publish your work in this journal}

Clinical Epidemiology is an international, peer-reviewed, open access, online journal focusing on disease and drug epidemiology, identification of risk factors and screening procedures to develop optimal preventative initiatives and programs. Specific topics include: diagnosis, prognosis, treatment, screening, prevention, risk factor modification, Submit your manuscript here: https://www.dovepress.com/clinical-epidemiology-journa
40. Statistics Sweden. Education of the Population. Örebro: Statistics Sweden; 2014.

41. Sjoberg C, Edward C, Fastbom J, et al. Association between multi-dose drug dispensing and quality of drug treatment - a register-based study. PLoS One. 2011;6(10):e26574.

42. Bell JS, Johnell K, Wimmer BC, Wiese MD. Multidose drug dispensing and optimising drug use in older people. Age Ageing. 2013;42(5):556-558.

43. Calderón-Larrañaga A, Vetrano DL, Onder G, et al. Assessing and measuring chronic multimorbidity in the older population: a proposal for its operationalization. J Gerontol A Biol Sci Med Sci. 2017;72(10):1417-1423.

44. Brookhart MA, Sturmer T, Glynn RJ, Rassen J, Schneeweiss S. Confounding control in healthcare database research: challenges and potential approaches. Med Care. 2010;48(6 Suppl):S114-S120.

45. Morin L, Vetrano DL, Rizzuto D, Calderón-Larrañaga A, Fastbom J, Johnell K. Choosing wisely? Measuring the burden of medications in older adults near the end of life: nationwide, longitudinal cohort study. Am J Med. 2017;130(8):927-936.e9.

46. Bronskill SE, Gill SS, Paterson JM, Bell CM, Anderson GM, Rochon PA. Exploring variation in rates of polypharmacy across long term care homes. J Am Med Dir Assoc. 2012;13(3):309.e15-309.e21.

47. Haasum Y, Fastbom J, Johnell K. Institutionalization as a risk factor for inappropriate drug use in the elderly: a Swedish nationwide registerbased study. Ann Pharmacother. 2012;46(3):339-346.

48. Onder G, Liperoti R, Fialova D, et al. Polypharmacy in nursing home in Europe: results from the SHELTER study. J Gerontol A Biol Sci Med Sci. 2012;67(6):698-704.

49. Bjerrum L, Rosholm JU, Hallas J, Kragstrup J. Methods for estimating the occurrence of polypharmacy by means of a prescription database. Eur J Clin Pharmacol. 1997;53(1):7-11.

50. Cooper JA, Cadogan CA, Patterson SM, et al. Interventions to improve the appropriate use of polypharmacy in older people: a Cochrane systematic review. BMJ Open. 2015;5(12):e009235.

51. Johansson T, Abuzahra ME, Keller S, et al. Impact of strategies to reduce polypharmacy on clinically relevant endpoints: a systematic review and meta-analysis. Br J Clin Pharmacol. 2016;82(2):532-548.

52. Cadogan CA, Ryan C, Hughes CM. Appropriate polypharmacy and medicine safety: when many is not too many. Drug Saf. 2016;39(2): 109-116.

53. Wallerstedt SM, Fastbom J, Johnell K, Sjöberg C, Landahl S, Sundström A. Drug treatment in older people before and after the transition to a multi-dose drug dispensing system - a longitudinal analysis. PLoS One. 2013;8(6):e67088.

54. Brownlee S, Chalkidou K, Doust J, et al. Evidence for overuse of medical services around the world. Lancet. 2017;390(10090):156-168.

55. Scott I, Jayathissa S. Quality of drug prescribing in older patients: is there a problem and can we improve it? Intern Med J. 2010;40(1):7-18.

56. Benetos A, Rossignol P, Cherubini A, et al. Polypharmacy in the aging patient. JAMA. 2015;314(2):170.

57. Holmes HM, Hayley DC, Alexander GC, Sachs GA. Reconsidering medication appropriateness for patients late in life. Arch Intern Med. 2006;166(6):605-609.

58. Tjia J, Briesacher BA, Peterson D, Liu Q, Andrade SE, Mitchell SL. Use of medications of questionable benefit in advanced dementia. JAMA Intern Med. 2014;174(11):1763-1771.

systematic reviews, risk and safety of medical interventions, epidemiology and biostatistical methods, and evaluation of guidelines, translational medicine, health policies and economic evaluations. The manuscript management system is completely online and includes a very quick and fair peer-review system, which is all easy to use. 